\title{
Review Article \\ Hydrogen Gas: A Novel Type of Antioxidant in Modulating Sexual Organs Homeostasis
}

\author{
Yaxing Zhang $\mathbb{D}^{1},{ }^{1}$ Haimei Liu $\mathbb{D},{ }^{1}$ Jinwen Xu $\mathbb{D},{ }^{1}$ Shuhui Zheng $\mathbb{D}^{2}$, and Lequan Zhou $\mathbb{D}^{1}$ \\ ${ }^{1}$ Department of Physiology, School of Basic Medical Sciences, Guangzhou University of Chinese Medicine, Guangzhou, \\ Guangdong, China \\ ${ }^{2}$ Research Center for Translational Medicine, The First Affiliated Hospital, Sun Yat-sen University, Guangzhou, Guangdong, China
}

Correspondence should be addressed to Lequan Zhou; lqzhou@gzucm.edu.cn

Received 25 August 2020; Revised 23 November 2020; Accepted 30 December 2020; Published 16 January 2021

Academic Editor: Patricia Morales

Copyright (c) 2021 Yaxing Zhang et al. This is an open access article distributed under the Creative Commons Attribution License, which permits unrestricted use, distribution, and reproduction in any medium, provided the original work is properly cited.

Sex is a science of cutting edge but bathed in mystery. Coitus or sexual intercourse, which is at the core of sexual activities, requires healthy and functioning vessels to supply the pelvic region, thus contributing to clitoris erection and vaginal lubrication in female and penile erection in male. It is well known that nitric oxide (NO) is the main gas mediator of penile and clitoris erection. In addition, the lightest and diffusible gas molecule hydrogen $\left(\mathrm{H}_{2}\right)$ has been shown to improve erectile dysfunction (ED), testis injuries, sperm motility in male, preserve ovarian function, protect against uterine inflammation, preeclampsia, and breast cancer in female. Mechanistically, $\mathrm{H}_{2}$ has strong abilities to attenuate excessive oxidative stress by selectively reducing cytotoxic oxygen radicals, modulate immunity and inflammation, and inhibit injuries-induced cell death. Therefore, $\mathrm{H}_{2}$ is a novel bioactive gas molecule involved in modulating sexual organs homeostasis.

\section{Introduction}

Sex is a fundamental pleasure and quality-of-life issue $[1,2]$. Sexual medicine represents one of the oldest medical specialties, and ancient civilizations had no qualms in discussing their sexual health and sexuality-an openness that has not pervaded through to modern life [3]. Although crucial to the survival of our species, human sex is clearly more complex than mere reproduction; sexual medicine is involved in endocrinology, gynecology, andrology, genetics, neurology, angiology, psychology, sociology, anthropology, and other related disciplines; human sex is cutting edge but bathed in mystery $[1,3,4]$. Traditionally, sexual intercourse or coitus, which is viewed as that female vagina receives the male erect penis, is at the core of sexual activities.

Coitus requires healthy and functioning vessels to supply the pelvic region in males and females, thus generating penile and clitoris erection and vaginal lubrication, respectively $[5,6]$. Erection is a neurovascular event modulated by psychological and hormonal factors $[5,6]$. Nitric oxide
(NO), which is recognized as the main mediator of penile and clitoris erection, is synthesized and released from adjacent nonadrenergic noncholinergic (NANC) nerve endings via neuronal nitric oxide synthase (nNOS) and/or endothelial cells (ECs) via endothelial nitric oxide synthase (eNOS) upon mental and sensory stimuli via spinal reflex $[5,7,8]$. Upon its release, NO diffuses locally into adjacent cavernosal and vascular smooth muscle cells and binds with its physiologic receptor, soluble guanylyl cyclase (sGC) [5, 9]. This binding results in an enzyme conformational change, resulting in the conversion of guanosine triphosphate (GTP) to $3^{\prime}, 5^{\prime}$-cyclic guanosine monophosphate (cGMP) $[5,7,10]$. cGMP activates cGMP-dependent protein kinase (PKG); PKG phosphorylates several molecular targets, such as inositol 1,4,5-triphosphate (IP3) receptor, IP3 receptor-associated PKG substrate (IRAG), phospholamban (PLB), and calciumactivated potassium $\left(\mathrm{BK}_{\mathrm{Ca}}\right)$ channel $[11,12]$; these result in decreasing free cytosolic calcium $\left(\mathrm{Ca}^{2+}\right)$ concentration through increased its uptake into intracellular stores, such as the sarcoplasmic reticulum and mitochondria, and through 
increased efflux/decreased influx of $\mathrm{Ca}^{2+}$ across the plasma membrane, finally, induce relaxation of the vascular and cavernosal smooth muscle cells $[5,7,13]$.

It is well established that the principal determinant of smooth muscle cell contraction is the concentration of intracellular free $\mathrm{Ca}^{2+}$, and myosin light chain (MLC) phosphorylation mediated by activated myosin light chain kinase (MLCK) in response to increased $\mathrm{Ca}^{2+}$ is the main pathway by which vasoconstrictor stimuli induce crossbridge cycling of myosin and actin filaments [14]. The secondary pathway for smooth muscle cell contraction that is not directly dependent on $\mathrm{Ca}^{2+}$ concentration, but rather mediating $\mathrm{Ca}^{2+}$ sensitization, is the RhoA/Rho kinase pathway [14]. In response to contractile stimuli, the small GTPase RhoA activates the downstream effector Rho kinase, which phosphorylates C kinase potentiated protein phosphatase inhibitor (CPI-17) and subsequently induces the interaction of CPI-17 with the catalytic subunit of myosin light chain phosphatase (MLCP), or phosphorylates the myosin-binding subunit (MBS, also known as MYPT1) of MLCP, thus inhibiting MLCP activity and promoting the phosphorylated state of the MLC and contraction [14-16]. Besides the removal of $\mathrm{Ca}^{2+}$ from the cytosol discussed above, PKG also induces the phosphorylation of GTPase RhoA to inhibit its activity, thus contributing to smooth muscle relaxation $[11,12]$; additionally, PKG can directly interact with the myosinbinding subunit of MLCP to activate MLCP, thus inducing cGMP-dependent dephosphorylation of MLC and initiating the NO-mediated vasodilatation $[11,12,17-19]$.

The resulting dilation of the cavernosal arterioles and sinuses results in increasing blood flow (driven by the force of the arterial blood pressure) and a subsequent rise in intracavernosal pressure, which subsequently activates a veno-occlusive mechanism to limit the outflow of blood and further increases the pressure inside the cavernosum $[8,20]$. The erectile response ensues as the force of the elevated pressure expands the outer tunica albuginea of the penis, resulting in the increased penile length and diameter characteristic of erection [8]. Thus, at the onset of sexual stimulation, neuronal NO induced by neuronal depolarization and endothelial NO largely generated in response to shear forces brought on by increased blood flow in the penis serve, respectively, as a neurotransmitter initiating the erectile process and as a paracrine factor sustaining the full physiologic response $[5,8]$. The molecular mechanisms for female clitoral engorgement and vaginal lubrication are similar to those for male penis erection [6].

The discovery of NO as a small signaling gasotransmitter led to the investigation of the biological roles of other endogenously derived gases, carbon monoxide (CO), hydrogen sulfide $\left(\mathrm{H}_{2} \mathrm{~S}\right)$, sulfur dioxide $\left(\mathrm{SO}_{2}\right)$, hydrogen $\left(\mathrm{H}_{2}\right)$, and methane $\left(\mathrm{CH}_{4}\right)$ in human body or in animals [21-33]. Among these gases, the effects of $\mathrm{H}_{2} \mathrm{~S}$ [34-38] and $\mathrm{CO}$ [39-41] on sexual function and dysfunction have been extensively investigated. Mechanistically, sGC/cGMP pathway acts as one of the common target of these gasotransmitters during regulating penis erection [21]. In 2013, the protective effect of $\mathrm{H}_{2}$ on erectile dysfunction (ED) has also been confirmed [42]. Besides its role in $\mathrm{ED}$, supplement of exogenous $\mathrm{H}_{2}$ has been shown to suppress testis injuries and improve sperm motility in male and also has the protective effects on female sexual organs. Therefore, the aim of this review is to summarize and discuss the effects and mechanisms of $\mathrm{H}_{2}$ in modulating sexual organs homeostasis, including the injury repair of sexual organs, fertility, and sexual function.

\section{Basic Characteristics of $\mathbf{H}_{2}$}

$\mathrm{H}_{2}$ has two different characteristics when compared with other five gases above: first, $\mathrm{H}_{2}$ is the lightest and diffusible gas molecule [43]; second, mammalian cells have no abilities to produce $\mathrm{H}_{2}$ due to lack of the functional hydrogenase genes [44]. The endogenous $\mathrm{H}_{2}$ in mammalian is mainly produced by hydrogenases-containing bacterial species located in gastrointestinal tracts (such as Firmicutes and Bacteroidetes), respiratory system (such as Pseudomonas and Acinetobacter), mouth and pharynx (such as Eubacterium), vagina (such as Clostridium species), and skin (such as Corynebacterium, Acinetobacter, and Streptococcus) [45]. $\mathrm{H}_{2}$ acts as a substrate for sulfate reducing bacteria, methanogenic bacteria, and acetic acid producing bacteria to utilize and support their energy metabolism [44, 46-48]. Therefore, the endogenous $\mathrm{H}_{2}$ levels in mammals are dependent on the balance between $\mathrm{H}_{2}$-producing fermentative bacteria and $\mathrm{H}_{2}$ consumers [44, 46-48]. In addition to being a source of energy for some bacteria, $\mathrm{H}_{2}$ has strong abilities to alleviate excessive oxidative stress basically by selectively reducing cytotoxic oxygen radicals, modulate immunity and inflammation, and suppress injuries-induced cell death [29, 30, 43, 49-52].

\section{3. $\mathrm{H}_{2}$ Modulates Sexual Organs Homeostasis in Male}

$\mathrm{H}_{2}$ is a novel bioactive gas molecule; it has essential roles in modulating male sexual organs homeostasis. Supplement of exogenous $\mathrm{H}_{2}$ has the protective effects on diabetes-related ED. Moreover, $\mathrm{H}_{2}$ attenuates numerous chemical, mechanical, and radiation damages-induced testicular injuries, modulates testosterone levels, and improves sperm quality (Figure 1, left).

3.1. $\mathrm{H}_{2}$ and Erectile Dysfunction. The penis is a vascular organ that is sensitive to changes in oxidative stress and systemic NO levels [9]. Vascular homeostasis maintenance is an active process, involved in the growth, migration, and death of vascular cells and activation of immune cells in vasculature, as well as the generation and degradation of extracellular matrix (ECM); all these coordinate with environmental cues to maintain the function of blood vessels [53]. $\mathrm{H}_{2}$ has strong abilities to suppress the excessive oxidative stress, thus maintaining vascular homeostasis and function, such as inhibiting abdominal aortic coarctation (AAC)- induced vascular hypertrophy and intimal hyperplasia in arterialized vein grafts in rats, decreasing blood pressure in monocrotaline-, $\mathrm{N}$ omega-Nitro-L-arginine methyl ester (L-NAME; NOS inhibitor)-, or chronic intermittent hypoxia-induced hypertension in rats [51, 54-57]. $\mathrm{H}_{2}$ also alleviates vascular dysfunction in spontaneous hypertensive rats (SHR) partially 

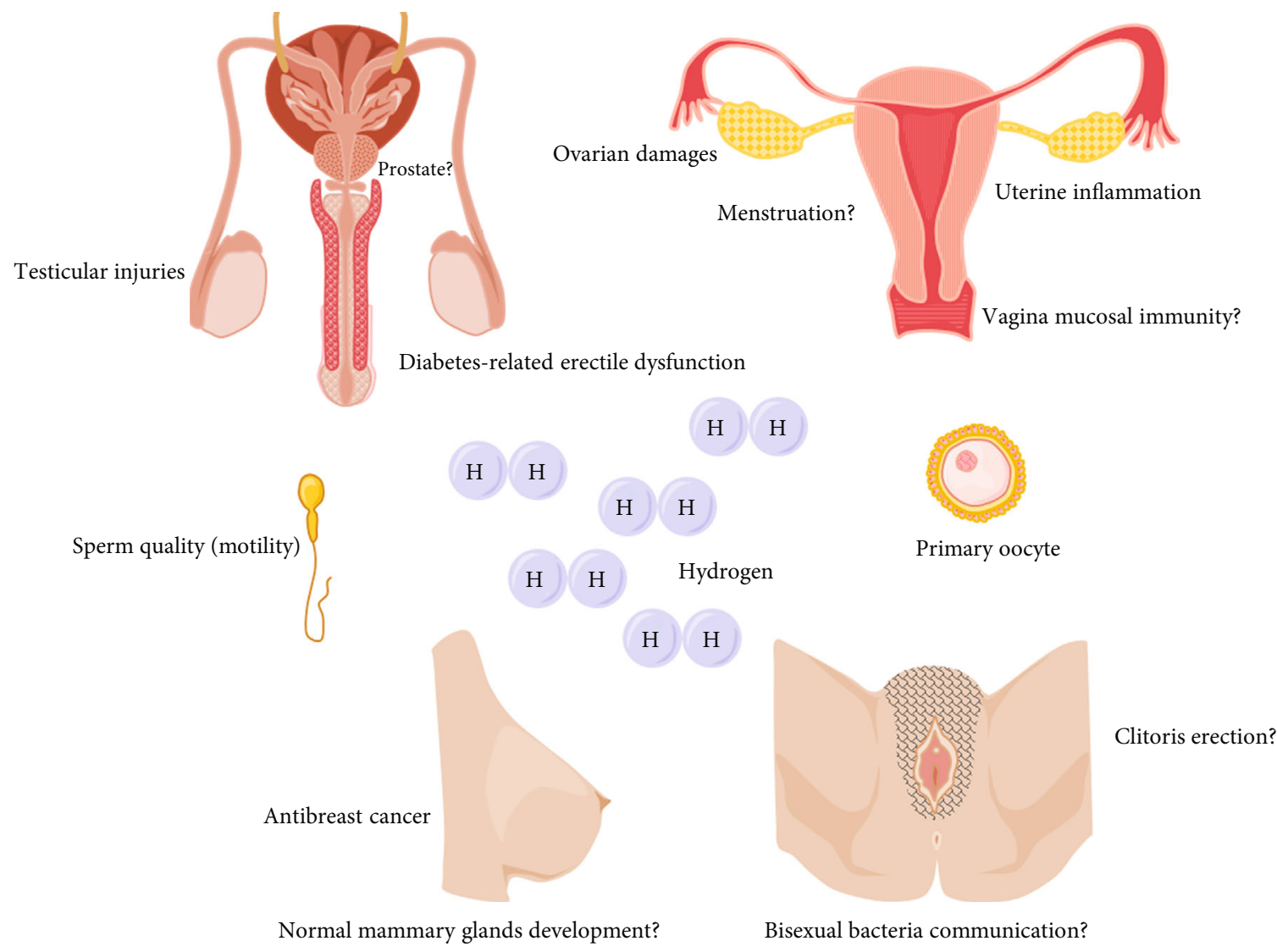

FIgURE 1: The target sex organs of $\mathrm{H}_{2}$ in animal models. In male animals, supplements of exogenous $\mathrm{H}_{2}$ may alleviate diabetes-related erectile dysfunction, various stresses-induced testicular injuries, and improve sperm quality (motility). In female animals, exogenous $\mathrm{H}_{2}$ has the protective effects on ovaries, uterus, and mammary glands, and improve the development of follicles. However, the functions of exogenous $\mathrm{H}_{2}$ in other sex organs, such as prostate in male, vagina and clitoris erection in female, the influence of bisexual bacteria communication in endogenous $\mathrm{H}_{2}$ levels, and the biological effects of exogenous $\mathrm{H}_{2}$ in sexual organs homeostasis, are not clear.

via enhancing NO bioavailability [58]. Fan et al. has investigated the effect of $\mathrm{H}_{2}$ on $\mathrm{ED}$ in a streptozotocin-induced diabetic rat model [42]. Compared with the diabetic rats, $\mathrm{H}_{2}$-rich saline gavage for 8 weeks improved ED, assessed by erectile frequency and intracavernous pressure measurement [42]. This protective effect of $\mathrm{H}_{2}$ on $\mathrm{ED}$ was related to reduce 8-hydroxy-2'-deoxyguanosine (8-OHdG) levels in serum and penis corpus cavernosum, attenuate malondialdehyde (MDA) levels and Bax expression in penis corpus cavernosum, elevate eNOS expression, NOS activity, NOx levels, and $\mathrm{Bcl}-2$ expression in penis corpus cavernosum [42]. Therefore, the beneficial effects of $\mathrm{H}_{2}$ on penile erection are primarily mediated by suppressing oxidative stress and apoptosis and inducing NO production in corpus cavernosum.

3.2. $\mathrm{H}_{2}$ and Testicular Toxicity of Smoking. Approximately $37 \%$ of male adults worldwide use tobacco, mainly cigarettes [59]. Smoking looks like relaxation; however, tobacco smoke contains more than 4000 kinds of chemical compounds, such as nicotine, heavy metals (cadmium and lead), and benzo(a)pyrene [60]. Substantial harmful effects of cigarette smoke on fecundity and reproduction have become apparent but are not generally appreciated [61]. Moreover, tobacco smoking is scientifically recognized as a risk factor of erectile impotence [60]. Oxidative stress and the resulting genetic and epi- genetic changes that result from smoking may correlate directly with reduced sperm function and reduced fertility $[60,62]$. Using cigarette smoke rat model, Chen et al. revealed that $\mathrm{H}_{2}$ subcutaneous injection increased the sperm count, increased serum testosterone levels, decreased the upregulation of sperm deformation rate and testicular MDA levels, and increased testicular and serum SOD activities [63]. Similarly, $\mathrm{H}_{2}$-rich saline decreased testicular and serum MDA levels, testicular $\mathrm{H}_{2} \mathrm{O}_{2}$, nitrotyrosine, and protein carbonyl levels; decreased testicular Caspase-3 activity; significantly increased both testicular and serum testosterone levels; and increased in sperm number and motility in mice subjected to chronic nicotine treatment [64]. However, whether $\mathrm{H}_{2}$ has the beneficial effects on cigarette smokerelated $\mathrm{ED}$ is not clear.

\section{3. $\mathrm{H}_{2}$ and Spinal Cord Hemisection-Induced Testicular} Injury. Most men with spinal cord injury (SCI) are infertile [65]. ED, ejaculatory dysfunction, and semen abnormalities contribute to the problem [65]. SCI is a highly inflammatory process that affects multiple organs that we do not fully understand, including the testis $[66,67]$. Moreover, reactive oxygen species (ROS) are higher in SCI men, and there is an inverse correlation between the level of ROS and sperm motility $[67,68]$. Ge et al. investigated the effects of $\mathrm{H}_{2}$-rich 
saline on the testicular biological function by establishing a hemi-sectioned spinal cord injury (hSCI) rat model (laminectomy at the T10-T12 level); they found that $\mathrm{H}_{2}$-rich saline upregulated the reduced ratio of testis weight/body weight, attenuated testicular morphological injury, improved the ultrastructural damage of cells in testis, increased the downregulated mean seminiferous tubular diameter and seminiferous epithelial thickness, reduced apoptosis of spermatogenic cells, increased testicular mitofusin-2 (an outer mitochondrial membrane GTPase involves in mitochondrial fusion and endoplasmic reticulum-mitochondria tethering [69]), and decreased testicular heme oxygenase-1 (HO-1, an enzyme catalyzes oxidation of heme to biologically active molecules: iron, a gene regulator; biliverdin, an antioxidant; and $\mathrm{CO}$, a heme ligand [70]) and high-mobility group box 1 (HMGB-1, an endogenous danger signal and inflammatory mediator) levels in rats subjected to hSCI [71]. However, the influences of $\mathrm{H}_{2}$ on the impaired erectile function after SCI still need further investigation.

3.4. $\mathrm{H}_{2}$ and Testicular Torsion. Testicular torsion is a true urological emergency most commonly seen in adolescence, which has been estimated to affect 1 in every 4000 males younger [72-74]. Testicular ischemia/reperfusion (I/R) caused by the twisting and release of the spermatic cord can result in biochemical and morphological changes; these have long-term effects on fertility and result in testicular atrophy, even if the testis can be salvaged $[72,73]$. The mechanism of testicular injury through reperfusion involves neutrophil recruitment, generation of ROS and reactive nitrogen species (RNS), proinflammatory cytokines and adhesion molecules, lipid peroxidation, apoptosis, anoxia, and alteration to microvascular blood flow $[72,73]$. Inhalation of $2 \% \mathrm{H}_{2}$ has the therapeutic effects on testicular I/R injury in rats as indicated by attenuating abnormal morphology and the impaired spermatogenesis, decreasing germ cell apoptosis and testicular MDA levels [75]. Moreover, $\mathrm{H}_{2}$-rich saline injection normalized lipid peroxidation levels and preserved activity of $\mathrm{SOD}$, thus reducing testicular I/R injury score and apoptosis index $[72,76]$. These studies confirmed the antioxidant, antiinflammatory, and antiapoptotic effects of $\mathrm{H}_{2}$ on testicular I/R injury.

\section{5. $\mathrm{H}_{2}$ and Testicular Damage Caused by Radiation. Nuclear} technologies utilization in power production, medicine, and industrial production drive the progress of modern civilization and make a better life; however, people exposed to ionizing radiation have the potential health threats $[77,78]$. Ionizing radiation induced oxidative stress, epigenetic changes and genomic instability, and disturbed mitochondria, thus inducing the detrimental effects on organisms [79-82]. As one of the most radiosensitive organs, testicular function can be significantly impaired by ionizing radiation exposure [83]. $\mathrm{H}_{2}$-rich saline attenuated $\gamma$-radiation-induced testis damage through reducing testicular hydroxyl radicals $(\cdot \mathrm{OH}), \mathrm{MDA}$, protein carbonyl, and $8-\mathrm{OHdG}$ levels, while restoring serum testosterone levels, and SOD and GSH levels in testis, attenuating apoptosis of spermatogenic cells, preserving stem spermatogonia survival, and the daily sperm production and sperm quality [78, 84]. This provides the evidence that $\mathrm{H}_{2}$ has potential clinical applications in preventing testicular damage caused by radiation.

3.6. $\mathrm{H}_{2}$ and Sperm Motility. The average life expectancy has been increasing, and many aging men have the need to maintain normal sexual function, as well as their fecundity [85]. Aging-related oxidative stress plays a crucial role in the progression of age-related male infertility [85]. As we have discussed above, $\mathrm{H}_{2}$ improved sperm quality after testicular damage in vivo. The protective effect of $\mathrm{H}_{2}$ on sperm motility was further confirmed by human sperm in vitro; it improved sperm motility of experimentally damaged sperm suspensions from patients left at room temperature for $>5$ days or frozen immediately after ejaculation, and increased mitochondrial membrane potential; however, $\mathrm{H}_{2}$ treatment did not affect sperm swimming speed [86]. Therefore, $\mathrm{H}_{2}$ is a new promising tool for male infertility treatments [86].

Varicocele-induced male infertility potentially involves oxidative stress [87]. Recently, Inagaki developed a siliconbased agent that produces $\mathrm{H}_{2}$ by the reaction with water [87-89]. By using this agent, they have investigated the therapeutic effects of $\mathrm{H}_{2}$ on a varicocele rat model. They found that oral intake of the silicon-based agent improves epididymal sperm motility and in vitro fertilization rates via $\mathrm{H}_{2}$ production and subsequent reduction of oxidative stress [87]. The protective effect of $\mathrm{H}_{2}$ on sperm was also confirmed in normal young and aged male mice. $\mathrm{Ku}$ et al. found that $\mathrm{H}_{2}$-rich water or Korean Red Ginseng treatment by gavage stimulated spermatogenesis followed by increasing sperm motility in 3month-old male mice and increasing sperm count and sperm motility in 12-month-old male mice [85]. These effects were strengthened synergistically by the $\mathrm{H}_{2}$-rich Korean Red Ginseng water mixture [85]. These functional water has the abilities to modulate the expressions of antioxidation (PPx3, PPx4, GSTm5, and GPx4), spermatogenesis (inhibin- $\alpha$, neptin-2 and cyclic AMP responsive element modulator (CREB)), antiaging (sirtuin 1 (SIRT1) and SIRT2), and angiogenesis (visfatin and vascular endothelial growth factor (VEGF)) related genes in the testes and decrease serum ROS level [85]. The serum testosterone levels were increased in both young and old mice after drinking 4 weeks of $\mathrm{H}_{2}$-rich water; the serum folliclestimulating hormone (FSH) levels were increased only in old mice receiving $\mathrm{H}_{2}$-rich water [85]. However, the levels of FSH as well as of luteinizing hormone (LH) were not significantly influenced in rats after 4 days of receiving a single dose of $\gamma$-irradiation or treatment with $\mathrm{H}_{2}$-rich saline [84]. The difference of these two studies might be related to the time and forms of $\mathrm{H}_{2}$ used, as 4 days are too short to influence hormones levels, and might also be related to the animal model used.

\section{4. $\mathrm{H}_{2}$ Modulates Sexual Organs Homeostasis in Female}

In female animal models, supplements of the exogenous $\mathrm{H}_{2}$ also display essential roles in modulating sexual organs homeostasis. $\mathrm{H}_{2}$ may alleviate I/R- and drug-induced ovarian injuries; improve postmenopausal osteoporosis, premature 
ovarian failure (POF), and follicles development; reduce uterine inflammation; ameliorate several characteristics of preeclampsia; and has the antibreast cancer effect (Figure 1, right).

4.1. $\mathrm{H}_{2}$ and Adnexal Torsion. Adnexal torsion is a gynecologic disorder caused by the partial or complete twist of the ovary and/or the fallopian tube on the axis created between the infundibulopelvic ligament and the utero-ovarian ligament $[90,91]$. It usually presents as a sudden, continuous, nonspecific pain in the lower abdomen, and the annual prevalence is approximately $2 \%$ to $6 \%[91,92]$. As that ischemia is the direct consequence of the twists in the adnexa, therefore, laparoscopic detorsion should be performed in order to preserve the integrity of the ovaries and fertility; nevertheless, detorsion creates I/R injury, which causes ovarian damage through the induction of oxidative stress, inflammation, and apoptosis [93-95]. $\mathrm{H}_{2}$-rich saline attenuated follicular injury, edema, hemorrhage, loss of cohesion, and the upregulation of apoptotic index in ovarian $\mathrm{I} / \mathrm{R}$ rats model via modulating $\mathrm{MDA}$ and glutathione-S-transferase (GST) levels [96]. Therefore, $\mathrm{H}_{2}$ is a novel bioactive gas molecule in attenuating I/R-induced ovary injury.

4.2. $\mathrm{H}_{2}$ and Osteoporosis after Menopause. Menopause is defined as the permanent cessation of ovulation and menstruation due to ovarian failure [97]. The menopause, as measured by the last menstrual period, occurs at an average age of 50.7 years [98]. The estrogen $\left(\mathrm{E}_{2}\right)$ level after menopause is inadequate to maintain $\mathrm{E}_{2}$-dependent tissues, leads to the gradually atrophy of breasts, vulvar and vaginal, thinning and dryness of the vaginal epithelium, and osteoporosis [99-101].

Oxidative stress plays an essential role in the progression of osteoporosis $[102,103]$. The animal study indicated that $\mathrm{H}_{2}$ has the beneficial effect on osteoporosis after menopause $[104,105]$. Daily treatment with $60 \% \mathrm{H}_{2}$ protected against postmenopausal osteoporosis in an ovariectomized (OVX) mice model by reducing serum levels of proinflammatory cytokine IL-1 $\beta$, IL-6, and TNF- $\alpha$ [105]. Moreover, $\mathrm{H}_{2}$-rich water consumption prevented osteopenia in OVX rats, while had no significant effect on plasma $E_{2}$ levels [104]. The plasma levels of NOx, which is the stable end products of $\mathrm{NO}$, and femur eNOS mRNA levels were increased by $\mathrm{H}_{2}$ in ovariectomized rats, indicated that $\mathrm{H}_{2}$ can induce NO production [104]. However, if $\mathrm{H}_{2}$ can improve atrophy of breasts, vulvar, and vaginal, and thinning and dryness of the vaginal epithelium after menopause are not clear.

4.3. $\mathrm{H}_{2}$ and Premature Ovarian Failure. Menopause before the age of 40 years is defined as premature menopause, also known as POF, which occurs in 1\% of women [106-111]. $\mathrm{POF}$ is characterized by amenorrhoea, hypo-oestrogenism, and elevated gonadotrophin levels due to cessation of ovarian function [106-110]. Genetic aberrations, autoimmune ovarian damage, latrogenic following surgical, radiotherapeutic or chemotherapeutic interventions as in malignancies, and environmental factors like viral infections and biohazardous environmental chemicals, etc., are responsible for the pathogenesis of POF [106, 112-116]. $\mathrm{H}_{2}$ has been shown to protect against $\mathrm{POF}$ and the drug-induced ovarian injury $[117,118]$. Drinking $\mathrm{H}_{2}$-rich water increased serum anti-Müllerian hormone (acting as a marker in POF and a good predictor of the time of menopause [119]) levels and ovarian Bcl-2 expression, decreased granulosa cell apoptotic index, and Bax/Bcl2 ratio in a POF mice model induced by immunized with zona pellucida glycoprotein 3 ; these indicated that $\mathrm{H}_{2}$ exerted protective effect on ovarian reserve function in mice with immune POF [117]. In a cisplatin-induced ovarian injury rat model, $\mathrm{H}_{2}$-rich saline attenuated the serum folliclestimulating hormone (FSH) release, elevated the serum level of $E_{2}$, improved the development of follicles, and reduced the damage to the ovarian cortex [118]. This protective effect of $\mathrm{H}_{2}$-rich saline on ovarian injury is involved in increasing the activities of SOD and catalase and reducing the level of MDA in the serum and ovarian tissue and increasing ovarian nuclear factor erythroid 2-related factor 2 (Nrf2) expression [118]. Future studies should focus on whether $\mathrm{H}_{2}$ can modulate the immune dysfunction in POF animal models.

4.4. $\mathrm{H}_{2}$ and Uterine Inflammation. Intrauterine inflammation causes preterm birth and is associated with complications in preterm neonates $[120,121]$. Nakano et al. found that drinking $\mathrm{H}_{2}$-rich water may significantly prevent uterine inflammation via reducing the expression of proinflammatory cytokines (Tnf and Il6), contractile-associated proteins (Cox2 (Ptgs2), Cx43 (Gja1), and Oxtr), and Et1 (Edn1) in the uterus, increases the levels of progesterone in the maternal serum, potentially extending the duration of pregnancy in a murine model of lipopolysaccharide (LPS)-induced preterm birth [121]. Their group also found that drinking $\mathrm{H}_{2}$ rich water ameliorates several characteristics of preeclampsia in the reduced uterine perfusion pressure (RUPP) rat model, such as decreasing mean arterial pressure, increasing fetal and placental weight, and attenuating angiogenic imbalance and oxidative stress [122]. Additionally, it is interesting to investigate the influence of $\mathrm{H}_{2}$ on menstruation.

4.5. $\mathrm{H}_{2}$ and Breast Cancer. Drinking the electrochemically reduced water, which is rich in $\mathrm{H}_{2}$, may delay mammary tumors growth in mice and inhibit the survival and induce apoptosis of human (MCF-7 and MDA-MB-453) and mouse (TUBO) breast cancer cell lines in vitro [123]. Another study indicated that combination of ammonia borane-mediated $\mathrm{H}_{2}$ therapy and polydopamine-mediated photothermal therapy may maximize the therapeutic effects on breast cancer in mice and overcome undesirable proinflammatory responses [124]. However, the effects of $\mathrm{H}_{2}$ on the normal growth and development of mammary glands are not clear.

\section{Perspective}

5.1. Gases Interaction in Modulating Sexual Organs Homeostasis. NO, $\mathrm{CO}$, and $\mathrm{H}_{2} \mathrm{~S}$ are key gas molecules contributing to penis erection $[5,34,35,39,41,125] . \mathrm{H}_{2} \mathrm{~S}$ and $\mathrm{H}_{2}$ can induce NO production in corpus cavernosum [42, 126]. Moreover, the exogenous $\mathrm{CO}$ can induce relaxation of 
phenylephrine precontracted corpus cavernosum smooth muscle in response to electrical field stimulation [39]. This relaxation may be mediated by CO-dependent activation of sGC and subsequent elevation of cGMP or CO-dependent induction of $\mathrm{NO}$ release $[39,127,128] . \mathrm{H}_{2}$ is primarily produced by hydrogenase-expressing fermentative bacteria in human body; $\mathrm{H}_{2} \mathrm{~S}$ and $\mathrm{CH}_{4}$ are the by-products of $\mathrm{H}_{2}$ metabolism derived from sulfate-reducing bacteria and methanogenic bacteria, respectively [44]. Therefore, the role of intestinal flora-derived $\mathrm{H}_{2} \mathrm{~S}$ should also be taken into consideration when discussing sex organs homeostasis. It is well known that mammalian cells can also produce $\mathrm{H}_{2} \mathrm{~S}$ and $\mathrm{CH}_{4}$ by its own enzyme system $[26,31,129]$. It is not clear whether exogenous or endogenous $\mathrm{H}_{2}$ can modulate $\mathrm{CO}$, $\mathrm{H}_{2} \mathrm{~S}$, or $\mathrm{CH}_{4}$ production in corpus cavernosum. $\mathrm{CH}_{4}$ has anti-inflammatory effects, and numerous studies have shown the involvement of inflammatory processes in male and female sexual dysfunction $[6,130]$. The function of $\mathrm{CH}_{4}$ in sexual dysfunction is not clear, if exist, whether the key molecular mechanism of $\mathrm{CH}_{4}$ is mediated by the classical $\mathrm{NO}, \mathrm{H}_{2} \mathrm{~S}$, or $\mathrm{CO}$ signal, or by other unknown targets? Is this effect influenced by the endogenous or exogenous $\mathrm{H}_{2}$ ? These endogenous gas molecules always exist as the gas mixture; they may arrive at the sexual organs by free diffusion or by blood circulation, thus influencing sexual organs homeostasis, respectively, together by another one or more. Therefore, there exist the gases interaction networks among $\mathrm{NO}, \mathrm{CO}$, $\mathrm{H}_{2} \mathrm{~S}, \mathrm{H}_{2}$, and $\mathrm{CH}_{4}$, and these networks might play the essential roles in modulating sexual organs homeostasis.

5.2. Interaction between Sexual Activities and Endogenous $\mathrm{H}_{2}$. The sexual activities between male and female always involve emotional communication, kissing and saliva exchange, petting, touching genitals, oral sex (fellatio (oral-penile contact), cunnilingus (oral-vaginal contact), and analingus (oral-anal contact)), anal sex, coitus, etc. [131-137]. Kissing and saliva exchange can transmit oral microbiota [138], and oral sex can also transmit oral, respiratory, and genital infections from one site in body to the other $[133,135]$. Human lower reproductive tract (vagina, cervix, and penile urethra) is the direct place for sexual intercourse; the mucosal epithelia in these organs are exposed to sexually transmitted microbes $[139,140]$. As we have discussed above, the endogenous $\mathrm{H}_{2}$ is mainly produced in the gastrointestinal tracts, mouth and pharynx, vagina, respiratory system, and skin by $\mathrm{H}_{2}$-producing fermentative bacteria [45]. Therefore, sexual activities will promote the bisexual bacteria communication, including the bacteria in the organs involved in sexual activities, and the endogenous $\mathrm{H}_{2}$ levels might be easily affected by sexual activities. However, the biological functions of this transfer in sex activities are not clear.

5.3. The Endogenous $\mathrm{H}_{2}$ in Sexual Organs Homeostasis. The endogenous $\mathrm{H}_{2}$ is the product of the metabolism of anaerobic bacteria $\left(\mathrm{H}_{2}\right.$-producing fermentative bacteria) by degrading the unabsorbed hydrocarbons in the intestinal tract of mammals $[30,44]$. Therefore, diet can influence the production of endogenous $\mathrm{H}_{2}$. For example, $\mathrm{H}_{2}$ was produced in greater amounts by breast-fed infants than by infants feeding with a soy-based or a milk-based formula, presumably the consequence of incomplete absorption of breast milk oligosaccharides [141]. Another clinical trial in Japan indicated that curcumin can activate carbohydrate colonic fermentation in human, thereby increasing the concentration of breath $\mathrm{H}_{2}$ [142]. In animals, lactulose, L-arabinose, and diet with $20 \%$ high amylose cornstarch can enhance the production of endogenous $\mathrm{H}_{2}$; the produced endogenous $\mathrm{H}_{2}$ is an essential regulator of liver homeostasis, such as improving hepatic I/R injury, liver regeneration, and hepatic steatosis as well as glucose and lipids homeostasis [44, 143-145]. The exogenous $\mathrm{H}_{2}$ has protective effects on testes damages and alleviates ED in a diabetic rat model; in female animal disease models, exogenous $\mathrm{H}_{2}$ has the antibreast cancer effects and protects ovaries and uterus against injuries (Figure 1). However, little is known about the physiological function of endogenous $\mathrm{H}_{2}$ in sexual organs homeostasis, such as penis and clitoral erection, and the influence of endogenous $\mathrm{H}_{2}$ in reproductive tract, for example, its roles in vagina, and mouth and pharynx mucosal immunity are also charming (Figure 1).

\section{Data Availability}

The data used to support the findings of this study are included within the article.

\section{Conflicts of Interest}

The authors declare that the research was conducted in the absence of any commercial or financial relationships that could be construed as a potential conflict of interest.

\section{Acknowledgments}

We apologize to all of the authors whose invaluable work we could not discuss or cite in this review due to space constraints. We should thank the Research Center for Integrative Medicine of Guangzhou University of Chinese Medicine (Key Laboratory of Chinese Medicine Pathogenesis and Therapy Research) for support. This work was supported by the National Natural Science Foundation of China (grant number 81900376 and 81901447), the Natural Science Foundation of Guangdong Province (grant number 2018A030313657 and 2018A030313733), and the Project funded by China Postdoctoral Science Foundation (grant number 2019M653238).

\section{References}

[1] J. R. Georgiadis, M. L. Kringelbach, and J. G. Pfaus, "Sex for fun: a synthesis of human and animal neurobiology," Nature Reviews Urology, vol. 9, no. 9, pp. 486-498, 2012.

[2] R. W. Stevenson, "Sexual medicine: why psychiatrists must talk to their patients about sex," Canadian Journal of Psychiatry, vol. 49, no. 10, pp. 673-677, 2004.

[3] A. Fenner, "Let's talk about sex...," Nature Reviews Urology, vol. 9, no. 9, p. 473, 2012.

[4] G. Wagner, "Sexual medicine in the medical curriculum," International Journal of Andrology, vol. 28, Supplement 2, pp. 7-8, 2005. 
[5] F. B. Priviero, R. Leite, R. C. Webb, and C. E. Teixeira, "Neurophysiological basis of penile erection," Acta Pharmacologica Sinica, vol. 28, no. 6, pp. 751-755, 2007.

[6] F. B. Calmasini, N. Klee, R. C. Webb, and F. Priviero, "Impact of immune system activation and vascular impairment on male and female sexual dysfunction," Sexual Medicine Reviews, vol. 7, no. 4, pp. 604-613, 2019.

[7] K. J. Allahdadi, R. C. Tostes, and R. C. Webb, "Female sexual dysfunction: therapeutic options and experimental challenges," Cardiovascular \& Hematological Agents in Medicinal Chemistry, vol. 7, no. 4, pp. 260-269, 2009.

[8] R. C. Tostes, F. S. Carneiro, A. J. Lee et al., "Cigarette smoking and erectile dysfunction: focus on $\mathrm{NO}$ bioavailability and ROS generation," The Journal of Sexual Medicine, vol. 5, no. 6, pp. 1284-1295, 2008.

[9] K. P. Nunes, H. Labazi, and R. C. Webb, "New insights into hypertension-associated erectile dysfunction," Current Opinion in Nephrology and Hypertension, vol. 21, no. 2, pp. 163-170, 2012.

[10] F. S. Carneiro, R. C. Webb, and R. C. Tostes, "Emerging Role for TNF- $\alpha$ in Erectile Dysfunction," Journal of Sexual Medicine, vol. 7, no. 12, pp. 3823-3834, 2010.

[11] C. S. Lin, G. Lin, and T. F. Lue, "Original research-basic science: cyclic nucleotide signaling in cavernous smooth muscle," Journal of Sexual Medicine, vol. 2, no. 4, pp. 478491, 2005.

[12] X. H. Wang, M. Tong, D. Dou, J. R. Usha, and Y. S. Gao, "Role of cGMP-dependent protein kinase in the cardiovascular system," Sheng Li Ke Xue Jin Zhan, vol. 36, no. 4, pp. 299303, 2005.

[13] L. Jin, A. E. Linder, T. M. Mills, and R. C. Webb, "Inhibition of the tonic contraction in the treatment of erectile dysfunction," Expert Opinion on Therapeutic Targets, vol. 7, no. 2, pp. 265-276, 2003.

[14] T. Szasz and R. C. Webb, "Rho-mancing to sensitize calcium signaling for contraction in the vasculature: role of rho kinase," Advances in Pharmacology, vol. 78, pp. 303-322, 2017.

[15] D. L. Lee, R. C. Webb, and L. Jin, "Hypertension and RhoA/Rho-kinase signaling in the vasculature: highlights from the recent literature," Hypertension, vol. 44, no. 6, pp. 796-799, 2004.

[16] R. H. Hilgers and R. C. Webb, "Molecular aspects of arterial smooth muscle contraction: focus on Rho," Experimental Biology and Medicine, vol. 230, no. 11, pp. 829-835, 2005.

[17] H. K. Surks, N. Mochizuki, Y. Kasai et al., "Regulation of myosin phosphatase by a specific interaction with cGMPdependent protein kinase I," Science, vol. 286, no. 5444, pp. 1583-1587, 1999.

[18] Q. Q. Huang, S. A. Fisher, and F. V. Brozovich, "Unzipping the role of myosin light chain phosphatase in smooth muscle cell relaxation," Journal of Biological Chemistry, vol. 279, no. 1, pp. 597-603, 2004.

[19] E. Lee, D. B. Hayes, K. Langsetmo, E. J. Sundberg, and T. C. Tao, "Interactions between the leucine-zipper motif of cGMP-dependent protein kinase and the C-terminal region of the targeting subunit of myosin light chain phosphatase," Journal of Molecular Biology, vol. 373, no. 5, pp. 1198-1212, 2007.

[20] T. M. Mills, R. W. Lewis, C. J. Wingard, K. Chitaley, and R. C. Webb, "Inhibition of tonic contraction-a novel way to approach erectile dysfunction," Journal of Andrology, vol. 23, no. 5, pp. S5-S9, 2002.

[21] G. Yetik-Anacak, R. Sorrentino, A. E. Linder, and N. Murat, "Gas what: NO is not the only answer to sexual function," British Journal of Pharmacology, vol. 172, no. 6, pp. 14341454, 2015.

[22] L. E. Otterbein, B. S. Zuckerbraun, M. Haga et al., "Carbon monoxide suppresses arteriosclerotic lesions associated with chronic graft rejection and with balloon injury," Nature Medicine, vol. 9, no. 2, pp. 183-190, 2003.

[23] L. E. Otterbein, R. Foresti, and R. Motterlini, "Heme oxygenase- 1 and carbon monoxide in the heart: the balancing act between danger signaling and pro-survival," Circulation Research, vol. 118, no. 12, pp. 1940-1959, 2016.

[24] A. L. True, M. Olive, M. Boehm et al., "Heme oxygenase-1 deficiency accelerates formation of arterial thrombosis through oxidative damage to the endothelium, which is rescued by inhaled carbon monoxide," Circulation Research, vol. 101, no. 9, pp. 893-901, 2007.

[25] C. Hine, E. Harputlugil, Y. Zhang et al., "Endogenous hydrogen sulfide production is essential for dietary restriction benefits," Cell, vol. 160, no. 1-2, pp. 132-144, 2015.

[26] G. Yang, L. Wu, B. Jiang et al., "H2S as a physiologic vasorelaxant: hypertension in mice with deletion of Cystathionine $\gamma$-lyase," Science, vol. 322, no. 5901, pp. 587-590, 2008.

[27] D. U. Shu-xu, H.-f. JIN, B. U. Ding-fang et al., "Endogenously generated sulfur dioxide and its vasorelaxant effect in rats," Acta Pharmacologica Sinica, vol. 29, no. 8, pp. 923-930, 2008.

[28] Y. Huang, C. Tang, J. Du, and H. Jin, "Endogenous sulfur dioxide: a new member of gasotransmitter family in the cardiovascular system," Oxidative Medicine and Cellular Longevity, vol. 2016, Article ID 8961951, 9 pages, 2016.

[29] I. Ohsawa, M. Ishikawa, K. Takahashi et al., "Hydrogen acts as a therapeutic antioxidant by selectively reducing cytotoxic oxygen radicals," Nature Medicine, vol. 13, no. 6, pp. 688694, 2007.

[30] Y. Zhang, S. Tan, J. Xu, and T. Wang, "Hydrogen therapy in cardiovascular and metabolic diseases: from bench to bedside," Cellular Physiology and Biochemistry, vol. 47, no. 1, pp. 1-10, 2018.

[31] M. Ghyczy, C. Torday, J. Kaszaki, A. Szabo, M. Czobel, and M. Boros, "Hypoxia-induced generation of methane in mitochondria and eukaryotic cells: an alternative approach to methanogenesis," Cellular Physiology and Biochemistry, vol. 21, no. 1-3, pp. 251-258, 2008.

[32] W. Liu, D. Wang, H. Tao, and X. Sun, "Is methane a new therapeutic gas?," Medical Gas Research, vol. 2, no. 1, p. 25, 2012.

[33] Y. Jia, Z. Li, C. Liu, and J. Zhang, "Methane medicine: a rising star gas with powerful anti-inflammation, antioxidant, and antiapoptosis properties," Oxidative Medicine and Cellular Longevity, vol. 2018, Article ID 1912746, 10 pages, 2018.

[34] B. Srilatha, P. G. Adaikan, L. Li, and P. K. Moore, "Hydrogen sulphide: a novel endogenous gasotransmitter facilitates erectile function," Journal of Sexual Medicine, vol. 4, no. 5, pp. 1304-1311, 2007.

[35] R. d'Emmanuele di Villa Bianca, R. Sorrentino, P. Maffia et al., "Hydrogen sulfide as a mediator of human corpus cavernosum smooth-muscle relaxation," Proceedings of the National Academy of Sciences, vol. 106, no. 11, pp. 45134518, 2009. 
[36] R. d'Emmanuele di Villa Bianca, R. Sorrentino, V. Mirone, and G. Cirino, "Hydrogen sulfide and erectile function: a novel therapeutic target," Nature Reviews Urology, vol. 8, no. 5, pp. 286-289, 2011.

[37] G. Yetik-Anacak, A. Dikmen, C. Coletta et al., "Hydrogen sulfide compensates nitric oxide deficiency in murine corpus cavernosum," Pharmacological Research, vol. 113, Part A, pp. 38-43, 2016.

[38] F. Aydinoglu and N. Ogulener, "The relaxant mechanisms of hydrogen sulfide in corpus cavernosum," in Methods in Molecular Biology, pp. 137-150, Springer, 2007.

[39] D. W. Kim, C. Zhao, M. K. Kim, and J. K. Park, "Direct effect of carbon monoxide on relaxation induced by electrical field stimulation in rat corpus cavernosum," Korean Journal of Urology, vol. 51, no. 8, pp. 572-578, 2010.

[40] Y. G. Guo, W. B. Qin, W. J. Song, and S. Q. Wang, "Effect of endogenous carbon monoxide on the smooth muscle function of dog penile corpus cavernosum in vitro," Zhonghua Nan Ke Xue, vol. 12, no. 8, pp. 685-688, 2006.

[41] M. Ushiyama, T. Morita, T. Kuramochi, S. Yagi, and S. Katayama, "Erectile dysfunction in hypertensive rats results from impairment of the relaxation evoked by neurogenic carbon monoxide and nitric oxide," Hypertension Research, vol. 27, no. 4, pp. 253-261, 2004.

[42] M. Fan, X. Xu, X. He et al., "Protective effects of hydrogenrich saline against erectile dysfunction in a streptozotocin induced diabetic rat model," Journal of Urology, vol. 190, no. 1, pp. 350-356, 2013.

[43] S. Tan, Z. Long, X. Hou et al., " $\mathrm{H}(2)$ protects against lipopolysaccharide-induced cardiac dysfunction via blocking TLR4-mediated cytokines expression," Frontiers in Pharmacology, vol. 10, p. 865, 2019.

[44] Y. Zhang, J. Xu, and H. Yang, "Hydrogen: an endogenous regulator of liver homeostasis," Frontiers in Pharmacology, vol. 11, p. 877, 2020.

[45] S. M. Ostojic, "Non-gut microbiota as a source of bioactive hydrogen," Postgraduate Medical Journal, vol. 93, no. 1097, p. 170, 2017.

[46] F. Carbonero, A. C. Benefiel, and H. R. Gaskins, "Contributions of the microbial hydrogen economy to colonic homeostasis," Nature Reviews Gastroenterology \& Hepatology, vol. 9, no. 9, pp. 504-518, 2012.

[47] N. Nakamura, H. C. Lin, C. S. McSweeney, R. I. Mackie, and H. R. Gaskins, "Mechanisms of microbial hydrogen disposal in the human colon and implications for health and disease," Annual Review of Food Science and Technology, vol. 1, pp. 363-395, 2010.

[48] P. G. Wolf, A. Biswas, S. E. Morales, C. Greening, and H. R. Gaskins, "H2 metabolism is widespread and diverse among human colonic microbes," Gut Microbes, vol. 7, no. 3, pp. 235-245, 2016.

[49] Y. Zhang, J. Xu, Z. Long et al., "Hydrogen $(\mathrm{H}(2))$ inhibits isoproterenol-induced cardiac hypertrophy via antioxidative pathways," Frontiers in Pharmacology, vol. 7, p. 392, 2016.

[50] Y. Zhang, Z. Long, J. Xu et al., "Hydrogen inhibits isoproterenol-induced autophagy in cardiomyocytes in vitro and in vivo," Molecular Medicine Reports, vol. 16, no. 6, pp. 8253-8258, 2017.

[51] Y.-X. Zhang, J.-T. Xu, X.-C. You et al., "Inhibitory effects of hydrogen on proliferation and migration of vascular smooth muscle cells via down-regulation of mitogen/activated pro- tein kinase and ezrin-radixin-moesin signaling pathways," The Chinese Journal of Physiology, vol. 59, no. 1, pp. 46-55, 2016.

[52] M. Yang, Y. Dong, Q. He et al., "Hydrogen: a novel option in human disease treatment," Oxidative Medicine and Cellular Longevity, vol. 2020, Article ID 8384742, 17 pages, 2020.

[53] Y. Zhang, J. Wang, Y. Ding et al., "Migrasome and tetraspanins in vascular homeostasis: concept, present, and future," Frontiers in Cell and Developmental Biology, vol. 8, p. 438, 2020.

[54] Q. Sun, T. Kawamura, K. Masutani et al., "Oral intake of hydrogen-rich water inhibits intimal hyperplasia in arterialized vein grafts in rats," Cardiovascular Research, vol. 94, no. 1, pp. 144-153, 2012.

[55] P. Guan, X.-. M. Lin, S.-. C. Yang et al., "Hydrogen gas reduces chronic intermittent hypoxia-induced hypertension by inhibiting sympathetic nerve activity and increasing vasodilator responses via the antioxidation," Journal of Cellular Biochemistry, vol. 120, no. 3, pp. 3998-4008, 2019.

[56] Y. Wang, L. Jing, X.-M. Zhao et al., "Protective effects of hydrogen-rich saline on monocrotaline-induced pulmonary hypertension in a rat model," Respiratory Research, vol. 12, p. 26, 2011.

[57] X. Yang, L. Guo, X. Sun, X. Chen, and X. Tong, "Protective effects of hydrogen-rich saline in preeclampsia rat model," Placenta, vol. 32, no. 9, pp. 681-686, 2011.

[58] H. Zheng and Y. S. Yu, "Chronic hydrogen-rich saline treatment attenuates vascular dysfunction in spontaneous hypertensive rats," Biochemical Pharmacology, vol. 83, no. 9, pp. 1269-1277, 2012.

[59] R. Sharma, A. Harlev, A. Agarwal, and S. C. Esteves, "Cigarette smoking and semen quality: a new meta-analysis examining the effect of the 2010 World Health Organization laboratory methods for the examination of human semen," European Urology, vol. 70, no. 4, pp. 635-645, 2016.

[60] J. B. Dai, Z. X. Wang, and Z. D. Qiao, "The hazardous effects of tobacco smoking on male fertility," Asian Journal of Andrology, vol. 17, no. 6, pp. 954-960, 2015.

[61] AAO Practice Committee of the American Society for Reproductive Medicine, Electronic address and M Practice Committee of the American Society for Reproductive, "Smoking and infertility: a committee opinion," Fertility and Sterility, vol. 110, no. 4, pp. 611-618, 2012.

[62] A. Harlev, A. Agarwal, S. O. Gunes, A. Shetty, and S. S. du Plessis, "Smoking and male infertility: an evidence-based review," The World Journal of Men's Health, vol. 33, no. 3, pp. 143-160, 2015.

[63] S. Chen and W. Jiang, "Effect of hydrogen injected subcutaneously on testicular tissues of rats exposed to cigarette smoke," International Journal of Clinical and Experimental Medicine, vol. 8, no. 4, pp. 5565-5570, 2015.

[64] S. Li, D. Lu, Y. Zhang, and Y. Zhang, "Long-term treatment of hydrogen-rich saline abates testicular oxidative stress induced by nicotine in mice," Journal of Assisted Reproduction and Genetics, vol. 31, no. 1, pp. 109-114, 2014.

[65] N. L. Brackett, C. M. Lynne, E. Ibrahim, D. A. Ohl, and J. Sonksen, "Treatment of infertility in men with spinal cord injury," Nature Reviews Urology, vol. 7, no. 3, pp. 162-172, 2010.

[66] X. Zhang, E. Ibrahim, J. P. de Rivero Vaccari et al., "Involvement of the inflammasome in abnormal semen quality of 
men with spinal cord injury," Fertility and Sterility, vol. 99, no. 1, pp. 118-124, 2013.

[67] R. Anderson, R. Moses, S. Lenherr, J. M. Hotaling, and J. Myers, "Spinal cord injury and male infertility-a review of current literature, knowledge gaps, and future research," Translational Andrology and Urology, vol. 7, Supplement 3, pp. S373-S382, 2018.

[68] O. F. Padron, N. L. Brackett, R. K. Sharma, C. M. Lynne, A. J. Thomas Jr., and A. Agarwal, "Seminal reactive oxygen species and sperm motility and morphology in men with spinal cord injury," Fertility and Sterility, vol. 67, no. 6, pp. 1115-1120, 1997.

[69] R. Filadi, D. Pendin, and P. Pizzo, "Mitofusin 2: from functions to disease," Cell Death \& Disease, vol. 9, no. 3, p. 330, 2018.

[70] M. D. Maines, "The heme oxygenase system: a regulator of second messenger gases," Annual Review of Pharmacology and Toxicology, vol. 37, pp. 517-554, 1997.

[71] L. Ge, L.-H. Wei, C.-Q. Du et al., "Hydrogen-rich saline attenuates spinal cord hemisection-induced testicular injury in rats," Oncotarget, vol. 8, no. 26, pp. 42314-42331, 2017.

[72] A. Fenner, "Basic research: rat studies untwist roles of protective agents in testicular ischaemia-reperfusion injury," Nature Reviews Urology, vol. 9, no. 6, p. 291, 2012.

[73] E. Karaguzel, M. Kadihasanoglu, and O. Kutlu, "Mechanisms of testicular torsion and potential protective agents," Nature Reviews Urology, vol. 11, no. 7, pp. 391-399, 2014.

[74] B. W. Baker, J. K. Surratt, and W. Run, "Missed testicular torsion presenting with male infertility and erectile dysfunction," Zhonghua Nan Ke Xue, vol. 11, no. 8, pp. 615-616, 2005.

[75] J.-W. Lee, J.-I. Kim, Y.-A. Lee et al., "Inhaled hydrogen gas therapy for prevention of testicular ischemia/reperfusion injury in rats," Journal of Pediatric Surgery, vol. 47, no. 4, pp. 736-742, 2012.

[76] D. Jiang, D. Wu, Y. Zhang, B. Xu, X. Sun, and Z. Li, "Protective effects of hydrogen rich saline solution on experimental testicular ischemia-reperfusion injury in rats," Journal of Urology, vol. 187, no. 6, pp. 2249-2253, 2012.

[77] B. N. Pandey, A. Kumar, P. Tiwari, and K. P. Mishra, "Radiobiological basis in management of accidental radiation exposure," International Journal of Radiation Biology, vol. 86, no. 8, pp. 613-635, 2010.

[78] Y. Chuai, F. Gao, B. Li et al., "Hydrogen-rich saline attenuates radiation-induced male germ cell loss in mice through reducing hydroxyl radicals," Biochemical Journal, vol. 442, no. 1, pp. 49-56, 2012.

[79] D. Einor, A. Bonisoli-Alquati, D. Costantini, T. A. Mousseau, and A. P. Moller, "Ionizing radiation, antioxidant response and oxidative damage: a meta-analysis," Science of The Total Environment, vol. 548-549, pp. 463-471, 2016.

[80] I. Szumiel, "Ionizing radiation-induced oxidative stress, epigenetic changes and genomic instability: the pivotal role of mitochondria," International Journal of Radiation Biology, vol. 91, no. 1, pp. 1-12, 2015.

[81] W. W. Kam and R. B. Banati, "Effects of ionizing radiation on mitochondria," Free Radical Biology and Medicine, vol. 65, pp. 607-619, 2013.

[82] P. A. Riley, "Free radicals in biology: oxidative stress and the effects of ionizing radiation," International Journal of Radiation Biology, vol. 65, no. 1, pp. 27-33, 1994.
[83] J. Ding, H. Wang, Z. B. Wu, J. Zhao, S. Zhang, and W. Li, "Protection of murine spermatogenesis against ionizing radiation-induced testicular injury by a green tea polyphenol," Biology of Reproduction, vol. 92, no. 1, p. 6, 2015.

[84] Z. Jiang, B. Xu, M. Yang, Z. Li, Y. Zhang, and D. Jiang, "Protection by hydrogen against gamma ray-induced testicular damage in rats," Basic \& Clinical Pharmacology \& Toxicology, vol. 112, no. 3, pp. 186-191, 2013.

[85] J. Y. Ku, M. J. Park, H. J. Park, N. C. Park, and B. S. Joo, "Combination of Korean Red Ginseng extract and hydrogen-rich water improves spermatogenesis and sperm motility in male mice," Chinese Journal of Integrative Medicine, vol. 26, no. 5, pp. 361-369, 2020.

[86] K. Nakata, N. Yamashita, Y. Noda, and I. Ohsawa, "Stimulation of human damaged sperm motility with hydrogen molecule," Medical Gas Research, vol. 5, no. 1, p. 2, 2015.

[87] Y. Inagaki, S. Fukuhara, R. Imamura et al., "Novel hydrogenproducing Si-based agent reduces oxidative stress, improves sperm motility and in vitro fertilization rate in varicocele," Andrology, 2020.

[88] M. Kawamura, R. Imamura, Y. Kobayashi et al., "Oral administration of Si-based agent attenuates oxidative stress and ischemia-reperfusion injury in a rat model: a novel hydrogen administration method," Frontiers in Medicine, vol. 7, p. 95, 2020.

[89] Y. Kobayashi, R. Imamura, Y. Koyama et al., "Renoprotective and neuroprotective effects of enteric hydrogen generation from Si-based agent," Scientific Reports, vol. 10, no. 1, p. $5859,2020$.

[90] O. Adeyemi-Fowode, K. A. McCracken, and N. J. Todd, "Adnexal torsion," Journal of Pediatric and Adolescent Gynecology, vol. 31, no. 4, pp. 333-338, 2018.

[91] K. J. Sasaki and C. E. Miller, "Adnexal torsion: review of the literature," Journal of Minimally Invasive Gynecology, vol. 21, no. 2, pp. 196-202, 2014.

[92] G. Oelsner and D. Shashar, "Adnexal torsion," Clinical Obstetrics and Gynecology, vol. 49, no. 3, pp. 459-463, 2006.

[93] C. Huchon and A. Fauconnier, "Adnexal torsion: a literature review," European Journal of Obstetrics \& Gynecology and Reproductive Biology, vol. 150, no. 1, pp. 8-12, 2010.

[94] A. S. Laganà, V. Sofo, F. M. Salmeri et al., "Oxidative stress during ovarian torsion in pediatric and adolescent patients: changing the perspective of the disease," International Journal of Fertility \& Sterility, vol. 9, no. 4, pp. 416423, 2016.

[95] A. Topcu, G. Balik, M. Atak, T. Mercantepe, H. A. Uydu, and L. Tumkaya, "An investigation of the effects of metformin on ovarian ischemia-reperfusion injury in rats," European Journal of Pharmacology, vol. 865, article 172790, 2019.

[96] N. Gokalp, A. C. Basaklar, K. Sonmez et al., "Protective effect of hydrogen rich saline solution on experimental ovarian ischemia reperfusion model in rats," Journal of Pediatric Surgery, vol. 52, no. 3, pp. 492-497, 2012.

[97] J. S. Laven, "Genetics of early and normal menopause," Seminars in Reproductive Medicine, vol. 33, no. 6, pp. 377-383, 2017.

[98] D. Goswami and G. S. Conway, "Premature ovarian failure," Hormone Research, vol. 68, no. 4, pp. 196-202, 1998.

[99] P. S. Larmo, B. Yang, J. Hyssala, H. P. Kallio, and R. Erkkola, "Effects of sea buckthorn oil intake on vaginal atrophy in postmenopausal women: a randomized, double-blind, 
placebo-controlled study," Maturitas, vol. 79, no. 3, pp. 316321, 2014.

[100] R. Kagan, R. S. Williams, K. Pan, S. Mirkin, and J. H. Pickar, "A randomized, placebo- and active-controlled trial of bazedoxifene/conjugated estrogens for treatment of moderate to severe vulvar/vaginal atrophy in postmenopausal women," Menopause, vol. 17, no. 2, pp. 281-289, 2010.

[101] A. A. Shargil, "Hormone replacement therapy in perimenopausal women with a triphasic contraceptive compound: a three-year prospective study," International Journal of Fertility, vol. 30, no. 1, pp. 18-28, 1985.

[102] G. Bonaccorsi, I. Piva, P. Greco, and C. Cervellati, "Oxidative stress as a possible pathogenic cofactor of post-menopausal osteoporosis: existing evidence in support of the axis oestrogen deficiency-redox imbalance-bone loss," Indian Journal of Medical Research, vol. 147, no. 4, pp. 341-351, 2018.

[103] V. Domazetovic, G. Marcucci, T. Iantomasi, M. L. Brandi, and M. T. Vincenzini, "Oxidative stress in bone remodeling: role of antioxidants," Clinical Cases in Mineral and Bone Metabolism, vol. 14, no. 2, pp. 209-216, 2017.

[104] J. D. Guo, L. Li, Y. M. Shi, H. D. Wang, and S. X. Hou, "Hydrogen water consumption prevents osteopenia in ovariectomized rats," British Journal of Pharmacology, vol. 168, no. 6, pp. 1412-1420, 2013.

[105] G. Wu, L. Pan, J. Sun, G. Chen, and S. Wang, "Hydrogen gas protects against ovariectomy-induced osteoporosis by inhibiting NF-kappaB activation," Menopause, vol. 26, no. 7, pp. 785-792, 2019.

[106] D. Goswami and G. S. Conway, "Premature ovarian failure," Human Reproduction Update, vol. 11, no. 4, pp. 391-410, 1998.

[107] L. T. Shuster, D. J. Rhodes, B. S. Gostout, B. R. Grossardt, and W. A. Rocca, "Premature menopause or early menopause: long-term health consequences," Maturitas, vol. 65, no. 2, pp. 161-166, 2010.

[108] J. L. Luborsky, P. Meyer, M. F. Sowers, E. B. Gold, and N. Santoro, "Premature menopause in a multi-ethnic population study of the menopause transition," Human Reproduction, vol. 18, no. 1, pp. 199-206, 2003.

[109] A. Hoek, J. Schoemaker, and H. A. Drexhage, "Premature ovarian failure and ovarian autoimmunity," Endocrine Reviews, vol. 18, no. 1, pp. 107-134, 1997.

[110] A. N. Shelling, "Premature ovarian failure," Reproduction, vol. 140, no. 5, pp. 633-641, 1998.

[111] R. Koothirezhi and S. Ranganathan, Postmenopausal syndrome, StatPearls, Treasure Island (FL), 2020.

[112] H. Shiina, T. Matsumoto, T. Sato et al., "Premature ovarian failure in androgen receptor-deficient mice," Proceedings of the National Academy of Sciences, vol. 103, no. 1, pp. 224229, 2006.

[113] M. Uda, C. Ottolenghi, L. Crisponi et al., "Foxl2 disruption causes mouse ovarian failure by pervasive blockage of follicle development," Human Molecular Genetics, vol. 13, no. 11, pp. 1171-1181, 2004.

[114] T. Matikainen, G. I. Perez, A. Jurisicova et al., "Aromatic hydrocarbon receptor-driven Bax gene expression is required for premature ovarian failure caused by biohazardous environmental chemicals," Nature Genetics, vol. 28, no. 4, pp. 355-360, 2001.

[115] D. J. Allingham-Hawkins, R. Babul-Hirji, D. Chitayat et al., "Fragile X premutation is a significant risk factor for prema- ture ovarian failure: the International Collaborative POF in Fragile X study-preliminary data," American Journal of Medical Genetics, vol. 83, no. 4, pp. 322-325, 1999.

[116] S. Caburet, V. A. Arboleda, E. Llano et al., "Mutant cohesin in premature ovarian failure," New England Journal of Medicine, vol. 370, no. 10, pp. 943-949, 2014.

[117] X. He, S. Y. Wang, C. H. Yin, T. Wang, C. W. Jia, and Y. M. $\mathrm{Ma}$, "Hydrogen-rich water exerting a protective effect on ovarian reserve function in a mouse model of immune premature ovarian failure induced by zona pellucida 3," Chinese Medical Journal, vol. 129, no. 19, pp. 2331-2337, 2016.

[118] X. Meng, H. Chen, G. Wang, Y. Yu, and K. Xie, "Hydrogen-rich saline attenuates chemotherapy-induced ovarian injury via regulation of oxidative stress," Experimental and Therapeutic Medicine, vol. 10, no. 6, pp. 2277-2282, 2015.

[119] A. Kruszynska and J. Slowinska-Srzednicka, "Anti-Mullerian hormone (AMH) as a good predictor of time of menopause," Menopausal Review, vol. 2, pp. 47-50, 2017.

[120] A. K. Boyle, S. F. Rinaldi, J. E. Norman, and S. J. Stock, "Preterm birth: inflammation, fetal injury and treatment strategies," Journal of Reproductive Immunology, vol. 119, pp. 6266, 2017.

[121] T. Nakano, T. Kotani, K. Imai et al., "Effect of molecular hydrogen on uterine inflammation during preterm labour," Biomedical Reports, vol. 8, no. 5, pp. 454-460, 2018.

[122] T. Ushida, T. Kotani, H. Tsuda et al., "Molecular hydrogen ameliorates several characteristics of preeclampsia in the Reduced Uterine Perfusion Pressure (RUPP) rat model," Free Radical Biology and Medicine, vol. 101, pp. 524-533, 2016.

[123] G. V. Frajese, M. Benvenuto, R. Mattera et al., "Electrochemically reduced water delays mammary tumors growth in mice and inhibits breast cancer cells survival in vitro," EvidenceBased Complementary and Alternative Medicine, vol. 2018, Article ID 4753507, 14 pages, 2018.

[124] C. Zhang, D.-W. Zheng, C.-X. Li et al., "Hydrogen gas improves photothermal therapy of tumor and restrains the relapse of distant dormant tumor," Biomaterials, vol. 223, article 119472, 2019.

[125] P. Hedlund, L. Ny, P. Alm, and K. E. Andersson, "Cholinergic nerves in human corpus cavernosum and spongiosum contain nitric oxide synthase and heme oxygenase," The Journal of Urology, vol. 164, 3, Part 1, pp. 868-875, 2000.

[126] J. Meng, P. Ganesan Adaikan, and B. Srilatha, "Hydrogen sulfide promotes nitric oxide production in corpus cavernosum by enhancing expression of endothelial nitric oxide synthase," International Journal of Impotence Research, vol. 25, no. 3, pp. 86-90, 2013.

[127] D. Morse, J. Sethi, and A. M. Choi, "Carbon monoxidedependent signaling," Critical Care Medicine, vol. 30, Supplement 1, pp. S12-S17, 2002.

[128] C. Thorup, C. L. Jones, S. S. Gross, L. C. Moore, and M. S. Goligorsky, "Carbon monoxide induces vasodilation and nitric oxide release but suppresses endothelial NOS," American Journal of Physiology-Renal Physiology, vol. 277, no. 6, pp. F882-F889, 1999.

[129] Z. Altaany, F. Moccia, L. Munaron, D. Mancardi, and R. Wang, "Hydrogen sulfide and endothelial dysfunction: relationship with nitric oxide," Current Medicinal Chemistry, vol. 21, no. 32, pp. 3646-3661, 2014. 
[130] M. Z. Poles, L. Juhasz, and M. Boros, "Methane and inflammation - a review (fight fire with fire)," Intensive Care Medicine Experimental, vol. 7, no. 1, p. 68, 2019.

[131] E. W. Hook 3rd and K. Bernstein, "Kissing, saliva exchange, and transmission of Neisseria gonorrhoeae," The Lancet Infectious Diseases, vol. 19, no. 10, pp. e367-e369, 2019.

[132] D. L. Romo, C. Garnett, A. P. Younger et al., "Social media use and its association with sexual risk and parental monitoring among a primarily Hispanic adolescent population," Journal of Pediatric and Adolescent Gynecology, vol. 30, no. 4, pp. 466-473, 2017.

[133] T. Kumar, G. Puri, K. Aravinda, N. Arora, D. Patil, and R. Gupta, "Oral sex and oral health: an enigma in itself," Indian Journal of Sexually Transmitted Diseases and AIDS, vol. 36, no. 2, pp. 129-132, 2015.

[134] R. Stein, A. Sardinha, and C. G. Araujo, "Sexual activity and heart patients: a contemporary perspective," Canadian Journal of Cardiology, vol. 32, no. 4, pp. 410-420, 2016.

[135] R. Saini, S. Saini, and S. Sharma, "Oral sex, oral health and orogenital infections," Journal of Global Infectious Diseases, vol. 2, no. 1, pp. 57-62, 2010.

[136] R. A. Shtarkshall, S. Carmel, D. Jaffe-Hirschfield, and A. Woloski-Wruble, "Sexual milestones and factors associated with coitus initiation among Israeli high school students," Archives of Sexual Behavior, vol. 38, no. 4, pp. 591-604, 2009.

[137] P. Santtila, I. Wager, K. Witting et al., "Discrepancies between sexual desire and sexual activity: gender differences and associations with relationship satisfaction," Journal of Sex \& Marital Therapy, vol. 34, no. 1, pp. 31-44, 2007.

[138] R. Kort, M. Caspers, A. van de Graaf, W. van Egmond, B. Keijser, and G. Roeselers, "Shaping the oral microbiota through intimate kissing," Microbiome, vol. 2, p. 41, 2014.

[139] C. Agostinis, A. Mangogna, F. Bossi, G. Ricci, U. Kishore, and R. Bulla, "Uterine immunity and microbiota: a shifting paradigm," Frontiers in Immunology, vol. 10, p. 2387, 2019.

[140] K. Kawana, J. Matsumoto, S. Miura et al., "Expression of CD1d and ligand-induced cytokine production are tissue specific in mucosal epithelia of the human lower reproductive tract," Infection and Immunity, vol. 76, no. 7, pp. 30113018, 2018

[141] T. Jiang, F. L. Suarez, M. D. Levitt, S. E. Nelson, and E. E. Ziegler, "Gas production by feces of infants," Journal of Pediatric Gastroenterology and Nutrition, vol. 32, no. 5, pp. 534-541, 2001.

[142] A. Shimouchi, K. Nose, M. Takaoka, H. Hayashi, and T. Kondo, "Effect of dietary turmeric on breath hydrogen," Digestive Diseases and Sciences, vol. 54, no. 8, pp. 17251729, 2009.

[143] L. Zhao, Y. Wang, G. Zhang, T. Zhang, J. Lou, and J. Liu, "L-Arabinose elicits gut-derived hydrogen production and ameliorates metabolic syndrome in C57BL/6J mice on high-fat-diet," Nutrients, vol. 11, no. 12, 2019.

[144] J. Yu, W. Zhang, R. Zhang, X. Ruan, P. Ren, and B. Lu, "Lactulose accelerates liver regeneration in rats by inducing hydrogen," journal of Surgical Research, vol. 195, no. 1, pp. 128-135, 2015.

[145] H. Tanabe, Y. Sasaki, T. Yamamoto, S. Kiriyama, and N. Nishimura, "Suppressive effect of high hydrogen generating high amylose cornstarch on subacute hepatic ischemiareperfusion injury in rats," Bioscience of Microbiota, Food and Health, vol. 31, no. 4, pp. 103-108, 2012. 